\title{
BIODIESEL DE MAMONA NO DIESEL INTERIOR E METROPOLITANO EM TRATOR AGRÍCOLA
}

\author{
RUBENS A. TABILE ${ }^{1}$, AFONSO LOPES ${ }^{2}$, MIGUEL J. DABDOUB ${ }^{3}$, \\ FELIPE T. DA CAMARA ${ }^{4}$, CARLOS E. A. FURLANI ${ }^{5}$, ROUVERSON P. DA SILVA ${ }^{6}$
}

RESUMO: A demanda de recursos energéticos pelos sistemas de produção, aliada à escassez dos combustíveis fósseis, tem motivado a produção do Biodiesel, que é um combustível obtido de fontes renováveis. O objetivo deste trabalho foi realizar dois ensaios: o primeiro dinâmico, para avaliar o desempenho operacional utilizando como parâmetro o consumo de combustível, e o segundo, estático, para mensurar a opacidade da fumaça (material particulado) do motor de um trator agrícola, operando com diesel metropolitano e interior misturados ao Biodiesel de mamona, em sete proporções. O trabalho foi conduzido no Departamento de Engenharia Rural da UNESP/Jaboticabal - SP. Os resultados mostraram que o tipo de diesel influenciou no consumo de combustível e na opacidade da fumaça, sendo o diesel metropolitano de melhor qualidade; observou-se, também, que à medida que a proporção de Biodiesel aumentou, o mesmo ocorreu para o consumo de combustível; entretanto, a opacidade da fumaça reduziu com o acréscimo de Biodiesel até B75.

PALAVRAS-CHAVE: biocombustível, desempenho operacional, mecanização agrícola, Ricinus communis L., opacidade da fumaça.

\section{MAMONA BIODIESEL IN INTERIOR AND METROPOLITAN DIESEL IN AGRICULTURAL TRACTOR}

\begin{abstract}
The demand for energy resources by production systems allied to scarcity of fossil fuels has driven the production of Biodiesel, a fuel produced from renewable sources. The purpose of this study was realize two tests, the first dynamics to assess the operational performance as a parameter of consumption of fuel, the second static to measure the smoke opacity (particulate material) from an engine of a farm tractor, operating with interior and metropolitan diesel mixed with castor beans Biodiesel in seven proportions. The tests were conducted in the Rural Engineering Department of UNESP/Jaboticabal - SP. The results showed that the kind of diesel influenced the consumption of fuel and smoke opacity, and the metropolitan diesel showed better quality, it was observed as well that as biodiesel proportion increased, consumption of fuel increased too, however, the opacity of smoke decreased with an increase of Biodiesel by B75.
\end{abstract}

KEYWORDS: bio-fuel, operational performance, agricultural mechanization, Ricinus communis L., smoke opacity.

\section{INTRODUÇÃO}

A previsível escassez de petróleo, aliada, à consciência ecológica, está levando pesquisadores a procurar novas fontes de energia e processos de combustão mais eficientes e menos poluentes. A partir da década de 1970, o governo brasileiro começou a tomar medidas para reduzir a dependência de petróleo importado do Oriente Médio, desenvolvendo programas de pesquisa em biocombustíveis (SCHLOSSER et al., 2004; PARENTE, 2003).

\footnotetext{
${ }^{1}$ Eng $^{\circ}$ Agrícola, Doutorando em Engenharia Mecânica, USP/São Carlos SP. Bolsista Capes. rubens.tabile@gmail.com

${ }^{2}$ Eng $^{\circ}$ Agrícola, Prof. Adjunto, Departamento de Engenharia Rural, UNESP, Jaboticabal - SP.

${ }^{3}$ Quimico, Prof. Departamento de Química, USP, Ribeirão Preto - SP.

${ }^{4}$ Eng $^{\circ}$ Agrônomo, Doutorando, Departamento de Engenharia Rural, UNESP, Jaboticabal - SP. Bolsista da CAPES.

${ }^{5}$ Eng ${ }^{\circ}$ Agrônomo, Prof. Adjunto, Departamento de Engenharia Rural, UNESP, Jaboticabal - SP. Bolsista de produtividade CNPq.

${ }^{6}$ Eng ${ }^{0}$ Agrícola, Prof. Doutor, Departamento de Engenharia Rural, UNESP, Jaboticabal - SP. Bolsista de Produtividade CNPq.

Recebido pelo Conselho Editorial em: 1ㅇ-2-2008

Aprovado pelo Conselho Editorial em: 16-5-2009
} 
Segundo o Ministério de Minas e Energia, no Brasil, a oferta interna de energia para o ano de 2006 foi dividida em fontes não renováveis $(54,9 \%)$ e renováveis $(45,1 \%)$ (BRASIL, 2007). Em 1996, o Brasil consumiu o total de 30,16 bilhões de litros de óleo diesel, comparado com o anuário de 2006, em que o consumo foi de 39,12 bilhões de litros, houve aumento de 29,7\%. Em decorrência da reserva provada de petróleo ser de 11,8 bilhões de barris e o atual ritmo de produção de 1,72 milhões de barris por dia, essa reserva será suficiente por mais 18,8 anos; considerando outras fontes potenciais, esse horizonte pode chegar a 24,7 anos (BRASIL, 2006a).

Atualmente, no Brasil, para o sistema rodoviário, são disponibilizados dois tipos de óleo diesel: o metropolitano (500 $\mathrm{mg} \mathrm{kg}^{-1}$ de enxofre), apresentando coloração dourada, utilizado nos grandes centros urbanos, e o interior $\left(2.000 \mathrm{mg} \mathrm{kg}^{-1}\right.$ de enxofre), apresentando coloração avermelhada devido à adição de corante, comercializado nas demais localidades. Essa medida foi tomada para reduzir a quantidade de gases poluentes emitidos na atmosfera em locais onde existe grande concentração de veículos automotores.

Nesse contexto, a produção de biocombustíveis, em especial o Biodiesel, tornou-se uma das formas mais eficientes de diversificar a matriz energética, contribuindo para a conservação do meio ambiente, em razão da redução de emissão de gases do efeito estufa, do desenvolvimento econômico, da otimização e da descentralização de investimentos e do desenvolvimento social por meio da geração de emprego e renda no campo (COSTA \& HOESCHL, 2006).

Uma das características do Biodiesel é a significativa percentagem de massa de oxigênio em sua composição, em torno de $11 \%$, o que representa menor poder energético, mas contribui para o aumento do número de cetano e reduz as concentrações de gases poluentes emitidos (MUÑOZ et al., 2004). Em experimento realizado, a utilização de Biodiesel de óleo de girassol reduziu significativamente os níveis de emissão dos poluentes monóxidos de carbono $(32,2 \%)$, dióxido de carbono $(4,8 \%)$, hidrocarbonetos totais $(31,0 \%)$ e material particulado $(41,0 \%)$, entretanto houve aumento do óxido de nitrogênio $(5,7 \%)$. As emissões de hidrocarbonetos totais e monóxido de carbono podem ser reduzidas ainda mais com a adequação dos parâmetros de injeção como ponto de injeção e taxa de dosagem (MAZIERO et al., 2006; XIANOMING et al., 2005).

Uma das oleaginosas eleitas pelos programas federal e estadual de Biodiesel para fornecer matéria-prima para a produção do Biodiesel, foi a mamona (Ricinus communis L.). Entretanto, devido à presença de aproximadamente $90 \%$ de ácido ricinoleico em sua composição, o Biodiesel de mamona é um dos ésteres de óleos vegetais mais viscosos. Apresenta viscosidade cinemática de 14,5 cSt, fora dos limites permitidos pela portaria da Agência Nacional do Petróleo (2,5 a 5,5 cSt); entretanto, misturas com até $40 \%$ apresentam valor de 5,2 cSt, dentro do limite especificado (MAIA et al., 2006; MURUGESAN et al., 2009).

Estudos para avaliar o desempenho operacional de motores utilizando Biodiesel não apontaram indícios de aumento de desgaste, comprometimento de potência ou redução na durabilidade, entretanto vários autores observaram aumento no consumo de combustível à medida que se aumenta a proporção de uso do biocombustível. Comparando B0 com B100, LOPES et al. (2004), ensaiando trator agrícola, verificaram que o consumo de combustível específico aumentou $11 \%$, entretanto não foram observadas anomalias no funcionamento. CAMARA (2004), em experimento similar, evidenciou que, a partir de B25, houve aumento no consumo específico, chegando a 22,0\%, relacionando B0 com B100. Na mesma linha de pesquisa, GROTTA et al. (2008) verificaram que o consumo de combustível horário aumentou 4,9\%, o consumo ponderal $13,0 \%$ e o consumo específico 11,4\%, confrontando B0 com B100.

Ao comparar B0 com B100, produzido a partir de distintas fontes, LOPES (2006) concluiu que o consumo horário volumétrico aumentou de 6,4 a $24,8 \%$, de acordo com o tipo de Biodiesel utilizado, apresentando média geral de 13,3\%; o consumo horário ponderal variou de 7,5 a 29,2\%, com média geral de 15,6\%, e o consumo específico teve aumento médio de $17 \%$. 
ANGELO (2006), em ensaio com dinamômetro, verificou que o consumo específico de combustível aumentou com a adição de Biodiesel, entretanto nem sempre resultou em tendência proporcional. CASTELLANELLI et al. (2008), em ensaio dinamométrico com varias faixas de rotação do motor, verificaram que acima de B50 houve queda no desempenho e aumento no consumo específico de forma gradual. Observou-se que, em certas faixas de rotação, ocorre combustão incompleta das misturas devido à má atomização pelo sistema de injeção, que está ligado a características construtivas do motor, e que a faixa de melhor desempenho varia de acordo com as características de cada motor.

Para auxiliar os fabricantes e os agricultores na solução de problemas em máquinas e implementos agrícolas, há necessidade de verificar o desempenho desses equipamentos e estabelecer condições de testes semelhantes àquelas em que estiveram sendo usados (MANTOVANI et al., 1999), assim como para avaliar a viabilidade do Biodiesel de mamona, tornase fundamental o conhecimento das características do funcionamento das máquinas com o determinado combustível.

O objetivo do presente trabalho foi realizar dois ensaios: o primeiro dinâmico, para avaliar o desempenho operacional, e o segundo estático, para mensurar a opacidade da fumaça (material particulado) do motor de um trator agrícola, funcionando com Biodiesel de mamona, adicionado em várias proporções, a dois tipos de diesel (metropolitano e interior).

\section{MATERIAL E MÉTODOS}

O trabalho foi conduzido no Laboratório de Máquinas e Mecanização Agrícola (LAMMA), do Departamento de Engenharia Rural, UNESP, Câmpus de Jaboticabal - SP. A área possui localização geodésica definida pelas coordenadas $21^{\circ} 15^{\prime}$ latitude sul e $48^{\circ} 18^{\prime}$ ' longitude oeste, com altitude média de $570 \mathrm{~m}$. A região apresenta temperatura média anual de $22,2^{\circ} \mathrm{C}$, umidade relativa média de $71 \%$ e pressão atmosférica de $94,3 \mathrm{kPa}$. O clima da região, segundo a classificação de Köeppen, é do tipo Aw, definido como tropical úmido com estação chuvosa no verão e seca no inverno.

O solo da área experimental foi classificado, segundo EMBRAPA (1999), como Latossolo Vermelho eutroférrico típico, textura argilosa, A moderado caulinítico vídreo, relevo suave ondulado, com declividade média de $3 \%$. O teor médio de água no solo, no período do ensaio, no perfil de 0-15 e 15-30 cm de profundidade, foi, respectivamente, 11,2 e 13,4\%.

Utilizou-se de dois tipos de óleo diesel, classificados de acordo com a resolução da ANP $\mathrm{N}^{\mathrm{o}}$ 15, de 17 de julho de 2006 (BRASIL, 2006b), como: óleo diesel interior, tendo quantidade de enxofre total máxima de $2.000 \mathrm{mg} \mathrm{kg}^{-1}$, advindo da cidade de Jaboticabal - SP, e óleo diesel metropolitano, tendo quantidade de enxofre total máxima de $500 \mathrm{mg} \mathrm{kg}^{-1}$, advindo da cidade de São Paulo - SP.

O Biodiesel utilizado no trabalho foi do tipo mamona etílico destilado, sendo o processo de produção e de fornecimento do biocombustível a cargo do LADETEL - Laboratório de Desenvolvimento de Tecnologias Limpas da USP - Ribeirão Preto, num sistema de parceria com a UNESP - Jaboticabal desde 2001.

O experimento foi dividido em duas etapas: a primeira dinâmica, para avaliar o desempenho do trator, e a segunda estática, para avaliar a opacidade da fumaça.

$\mathrm{O}$ ensaio de desempenho teve delineamento experimental inteiramente casualizado, em esquema fatorial $2 \times 2 \times 7$, com três repetições, totalizando 84 observações. As combinações dos fatores foram dois tipos de diesel (interior e metropolitano), duas cargas na barra de tração (13 e $23 \mathrm{kN}$ ) e sete proporções de mistura diesel/Biodiesel (B0; B5; B15; B25; B50; B75 e B100). Cada parcela experimental possuía $30 \mathrm{~m}$ de comprimento e, entre si, na direção longitudinal, foi reservado espaço de $15 \mathrm{~m}$ destinado à realização de manobras, tráfego de máquinas e estabilização do conjunto motomecanizado em cada tratamento. 
No ensaio de opacidade, foi utilizado o delineamento inteiramente casualizado, em esquema fatorial $2 \times 7$, com três repetições, totalizando 14 observações. As combinações dos fatores foram dois tipos de diesel (interior e metropolitano) e sete proporções de mistura diesel/Biodiesel (B0; B5; B15; B25; B50; B75 e B100).

\section{Avaliação de desempenho}

O trator de teste utilizado foi da marca Valtra, modelo BM 100, 4x2 TDA, com potência de $73,6 \mathrm{~kW}(100 \mathrm{cv})$ no motor, a $2.350 \mathrm{rpm}$, massa total de $5.400 \mathrm{~kg}$, distribuídos 40 e $60 \%$ nos eixos dianteiro e traseiro, respectivamente, ressaltando-se que, durante os ensaios, o trator trabalhou na rotação de regime de $2.000 \mathrm{rpm}$. O trator encontrava-se instrumentado com célula de carga, medidor da rotação da tomada de potência (TDP), medidor da rotação dos rodados, unidade de radar e medidor da temperatura e do fluxo de combustível da entrada e do retorno da bomba injetora. Toda a instrumentação está ligada a um sistema de aquisição de dados, descrita conforme LOPES (2006).

Com a finalidade de oferecer carga à barra de tração do trator, foi utilizado um escarificador da marca Marchesan, modelo AST/MATIC 7, regulado com cinco hastes, com espaçamento entre hastes de $45 \mathrm{~cm}$, ponteira sem asa com $8 \mathrm{~cm}$ de largura, equipado com rolo destorroador e discos de corte de palha. A operação foi realizada com a haste do escarificador em duas profundidades de trabalho, 15 e $30 \mathrm{~cm}$, sendo a área manejada no sistema de plantio direto.

Devido à grande variabilidade da resistência do solo para a avaliação do consumo de combustível, e depois de conhecida a força na barra de tração requerida pelo escarificador, esse foi substituído por um segundo trator, denominado trator de frenagem, ensaiado na mesma condição de solo. Esse foi acoplado ao trator de teste por meio de cabo de aço, formando um comboio, e, em virtude da combinação de marcha no trator de frenagem, o esforço na barra de tração foi semelhante à escarificação a 15 e a $30 \mathrm{~cm}$ de profundidade. A velocidade de deslocamento média durante o ensaio foi $5,4 \mathrm{~km} \mathrm{~h}^{-1} \pm 0,2$ e a patinagem média dos rodados foi $8,6 \% \pm 0,4$.

O trator de frenagem utilizado foi da marca Valtra, modelo BH140, 4x2 TDA, com potência de $103 \mathrm{~kW}(140 \mathrm{cv})$ a $2.400 \mathrm{rpm}$ no motor, massa total de $7.600 \mathrm{~kg}$, distribuídos 40 e $60 \%$ nos eixos dianteiro e traseiro, respectivamente.

O consumo de combustível foi medido em cada parcela, obtendo-se o volume e a temperatura do combustível que alimenta a bomba injetora, e o volume e a temperatura do combustível que retorna ao tanque, sendo o combustível consumido dado pela diferença entre as duas medidas. A temperatura do combustível medida foi utilizada para a correção da densidade do combustível.

O sistema de medição de combustível consiste em dois conjuntos de sensores, um para a alimentação e o outro para o retorno da bomba injetora. Cada conjunto contém um medidor de fluxo da marca Oval Corporation, modelo Flowmate LSN 48, com precisão de 1\% sobre a vazão nominal e vazão máxima de $100 \mathrm{~L} \mathrm{~h}^{-1}$, e um sensor de temperatura do tipo resistivo, modelo PT 100 (resistência de $100 \mathrm{ohms} \mathrm{a} 0{ }^{\circ} \mathrm{C}$ ), com faixa de medida de $-200{ }^{\circ} \mathrm{C}$ a $800{ }^{\circ} \mathrm{C}$. Os valores de fluxo de combustível foram obtidos em $\mathrm{mL} \mathrm{s}^{-1}$, e a temperatura, em ${ }^{\circ} \mathrm{C}$.

Com base no volume consumido e no tempo de percurso em cada parcela, foi determinado o consumo horário volumétrico, conforme a eq. (1):

$$
\mathrm{Chv}=\left(\frac{\mathrm{Va}-\mathrm{Vr}}{\mathrm{t}}\right) 3,6
$$

em que,

Chv - consumo horário volumétrico, $\mathrm{L} \mathrm{h}^{-1}$;

$\mathrm{Va}$ - volume do combustível de alimentação, mL;

$\mathrm{Vr}$ - volume do combustível de retorno, $\mathrm{mL}$;

$\mathrm{t}$ - tempo de percurso na parcela, $\mathrm{s}$, e

3,6 - fator de conversão. 
Para calcular o consumo de combustível em massa, foi necessário determinar a densidade do combustível em função da temperatura. Nesse procedimento, o intervalo de temperatura foi de 10 a $70{ }^{\circ} \mathrm{C}$, avaliando a densidade em intervalos de $5{ }^{\circ} \mathrm{C}$, conforme eqs. (2) e (3):

$$
\begin{aligned}
& \text { Dint }=-0,0095 \mathrm{~T}^{2}+0,00173 \mathrm{P}^{2}-0,0124 \mathrm{~T}+0,5723 \mathrm{P}-0,0016 \mathrm{~T} \mathrm{P}+851 \\
& \text { Dmet }=-0,00965 \mathrm{~T}^{2}+0,00223 \mathrm{P}^{2}-0,00084 \mathrm{~T}+0,581 \mathrm{P}-0,00161 \mathrm{~T} \mathrm{P}+845 \\
& \mathrm{R}^{2}=0,98 \text { (ambas as equações). }
\end{aligned}
$$

em que,

Dint - densidade da mistura diesel interior e Biodiesel, $\mathrm{g} \mathrm{L}^{-1}$;

Dmet - densidade da mistura diesel metropolitano e Biodiesel, $\mathrm{g} \mathrm{L}^{-1}$;

$\mathrm{T}$ - temperatura do combustível, ${ }^{\circ} \mathrm{C}$;

$\mathrm{P}$ - proporção de Biodiesel, \%, e

$\mathrm{R}^{2}$ - coeficiente de determinação das regressões.

Para o cálculo do consumo horário ponderal, considerou-se a influência da temperatura no momento do teste para corrigir a densidade, conforme eq.(4):

$$
\text { Chp }=\frac{(\text { Va Dca }- \text { Vr Dcr })}{\mathrm{t}} 0,0036
$$

em que,

Chp - consumo horário ponderal, $\mathrm{kg} \mathrm{h}^{-1}$;

$\mathrm{Va}$ - volume do combustível de alimentação, mL;

Dca - densidade do combustível de alimentação, $\mathrm{g} \mathrm{L}^{-1}$

$\mathrm{Vr}$ - volume do combustível de retorno, $\mathrm{mL}$;

Dcr - densidade do combustível de retorno, $\mathrm{g} \mathrm{L}^{-1}$;

$\mathrm{t}$ - tempo de percurso na parcela, s, e

0,0036 - fator de conversão.

Consumo específico é o consumo de combustível expresso em unidade de massa por unidade de potência requerida na barra de tração, conforme eq. (5):

$$
\mathrm{Ce}=\left(\frac{\mathrm{Chp}}{\mathrm{PB}}\right) 1.000
$$

em que,

Ce - consumo específico, $\mathrm{g} \mathrm{kW} \mathrm{h}^{-1}$;

Chp - consumo horário ponderal, $\mathrm{kg} \mathrm{h}^{-1}$;

PB - potência na barra de tração, $\mathrm{kW}$, e

1.000 - fator de conversão.

\section{Avaliação de opacidade}

A opacidade da fumaça do motor do trator foi medida por um opacímetro de absorção de luz com fluxo parcial, da marca Tecnomotor, modelo TM 133, que está compatível com as normas NBR 13037, Inmetro e CEE 72/306.

Os ensaios foram realizados de acordo com o método da aceleração livre, que é o regime em que o motor é submetido ao máximo débito de combustível. As medições de opacidade foram feitas em K, que é o coeficiente de absorção de luz, e tem como unidade $\mathrm{m}^{-1}$ (TECNOMOTOR, 2001).

Ao término de cada determinação, realizou-se a drenagem completa do sistema de alimentação, evitando, com isso, a contaminação do ensaio seguinte. Além disso, depois de trocado 
o combustível, o motor ficou em funcionamento em torno de dez minutos antes do início de cada teste.

Os dados das duas etapas do experimento foram submetidos à análise de variância e ao teste de comparação de médias de Tukey, a 5\% de probabilidade (BANZATTO \& KRONKA, 2006). Para o consumo de combustível, foi estudado um modelo de ajuste de regressão que melhor explicasse o comportamento do mesmo, em função da proporção de Biodiesel, utilizando-se da análise de variância (Teste F) para selecionar o modelo de equação de maior expoente significativo.

\section{RESULTADOS E DISCUSSÃO}

Os resultados para as variáveis analisadas estão apresentados na Tabela 1.

TABELA 1. Síntese da análise de variância e teste de médias para consumo horário volumétrico (Chv), consumo horário ponderal (Chp), consumo específico (Ce) e opacidade da fumaça. Summary of analysis of variance and mean test for volumetric time consumption (Chv), weight hourly consumption (Chp), specific consumption (Ce) and smoke opacity.

\begin{tabular}{|c|c|c|c|c|}
\hline Fatores & $\begin{array}{l}\mathrm{Chv} \\
\mathrm{Lh}^{-1}\end{array}$ & $\begin{array}{c}\mathrm{Chp} \\
\mathrm{kg} \mathrm{h}^{-1}\end{array}$ & $\begin{array}{c}\mathrm{Ce} \\
\mathrm{g} \mathrm{kW} \mathrm{h}^{-1}\end{array}$ & $\begin{array}{c}\text { Opacidade } \\
\mathrm{m}^{-1}\end{array}$ \\
\hline \multicolumn{5}{|l|}{$\overline{\text { Diesel (D) }}$} \\
\hline Interior & $12,25 \quad \mathrm{~B}$ & $10,69 \mathrm{~B}$ & $416,4 \quad \mathrm{~B}$ & 0,96 \\
\hline Metropolitano & $11,95 \mathrm{~A}$ & $10,31 \mathrm{~A}$ & $397,8 \mathrm{~A}$ & 0,90 \\
\hline \multicolumn{5}{|l|}{ Proporção de Biodiesel (B) } \\
\hline$\overline{\mathrm{B} 0}$ & $11,33 \mathrm{~A}$ & 9,21 & $352,5 \mathrm{~A}$ & 1,02 \\
\hline B5 & $11,58 \mathrm{~A}$ & 9,51 & $367,8 \mathrm{~A}$ & 0,99 \\
\hline B15 & $11,85 \mathrm{AB}$ & 9,88 & $385,3 \mathrm{AB}$ & 0,94 \\
\hline B25 & $11,81 \mathrm{AB}$ & 10,00 & $387,2 \mathrm{AB}$ & 0,87 \\
\hline B50 & $12,43 \quad \mathrm{BC}$ & 10,92 & $417,5 \quad \mathrm{BC}$ & 0,89 \\
\hline B75 & $12,75 \quad \mathrm{C}$ & 11,62 & $451,8 \quad \mathrm{CD}$ & 0,84 \\
\hline $\mathrm{B} 100$ & $12,98 \quad \mathrm{C}$ & 12,33 & 487,5 & 0,95 \\
\hline \multicolumn{5}{|l|}{ Carga na barra $(\mathrm{C})$} \\
\hline $13 \mathrm{kN}$ & $10,32 \mathrm{~A}$ & 8,96 & $456,2 \quad \mathrm{~B}$ & -- \\
\hline $23 \mathrm{kN}$ & $13,89 \quad \mathrm{~B}$ & 12,03 & $358,0 \mathrm{~A}$ & -- \\
\hline \multicolumn{5}{|l|}{ Teste F } \\
\hline$\overline{\text { Diesel (D) }}$ & $6,79 *$ & $13,77 * *$ & $5,98 *$ & $40,06 * *$ \\
\hline Proporção de Biodiesel (B) & $16,96 * *$ & $74,49 * *$ & $22,93 * *$ & $26,27 * *$ \\
\hline Carga na barra $(\mathrm{C})$ & $977,82 * *$ & $916,28 * *$ & $166,38 * *$ & -- \\
\hline$D \times B$ & $0,82 \mathrm{~ns}$ & $0,78 \mathrm{~ns}$ & $0,91 \mathrm{~ns}$ & $4,06 * *$ \\
\hline $\mathrm{D} \times \mathrm{C}$ & $0,33 \mathrm{~ns}$ & $0,19 \mathrm{~ns}$ & $1,14 \mathrm{~ns}$ & -- \\
\hline$B \times C$ & $1,59 \mathrm{~ns}$ & $3,90 *$ & $0,84 \mathrm{~ns}$ & -- \\
\hline $\mathrm{D} \times \mathrm{B} \times \mathrm{C}$ & $0,42 \mathrm{~ns}$ & $0,42 \mathrm{~ns}$ & $0,55 \mathrm{~ns}$ & -- \\
\hline Média Geral & 12,10 & 10,50 & 407,1 & 0,93 \\
\hline C.V. \% & 4,32 & 4,42 & 8,58 & 3,35 \\
\hline
\end{tabular}

Médias seguidas pela mesma letra maiúscula na coluna não diferem entre si, pelo teste de Tukey, a 5\% de probabilidade; quando desacompanhada de letras, implica interações significativas, necessitando de quadro de desdobramento. **: significativo ( $\mathrm{P}<0,01)$; *: significativo $(\mathrm{P}<0,05)$; ns: não significativo; C.V.: coeficiente de variação.

\section{Consumo horário volumétrico}

Pela Tabela 1, observa-se que o diesel metropolitano apresentou menor consumo em relação ao diesel interior, sendo a redução média de $2,5 \%$ em função da qualidade desse produto. Observou-se que, na proporção de Biodiesel, não houve diferença significativa até B25, todavia, comparando B0 e B100, o consumo aumentou 14,6\% (Figura 1). De acordo com CASTELLANELLI et al. 
(2008), LOPES (2006) e MURUGESAN et al. (2009), esse aumento pode ser em função do menor poder calorífico do Biodiesel em relação ao diesel.

Conforme a Tabela 1, para o fator carga na barra, observa-se que, de 13 para $23 \mathrm{kN}$, o acréscimo foi de $80 \%$, entretanto esse provocou aumento de $34,6 \%$ no consumo, por isso recomenda-se, sempre que possível, utilizar a máxima capacidade de tração do trator, buscando maior eficiência. Na Figura 1, apresenta-se a análise de regressão linear do consumo volumétrico em função da proporção de mistura, e no eixo auxiliar, o aumento do consumo em percentagem com relação a B0.

Os resultados do presente trabalho condizem com os encontrados por DORADO et al. (2003) e REIS et al. (2006), ressaltando-se que o consumo volumétrico é a informação mais utilizada em meios não científicos para expressar consumo de combustível, inclusive no meio agrícola.

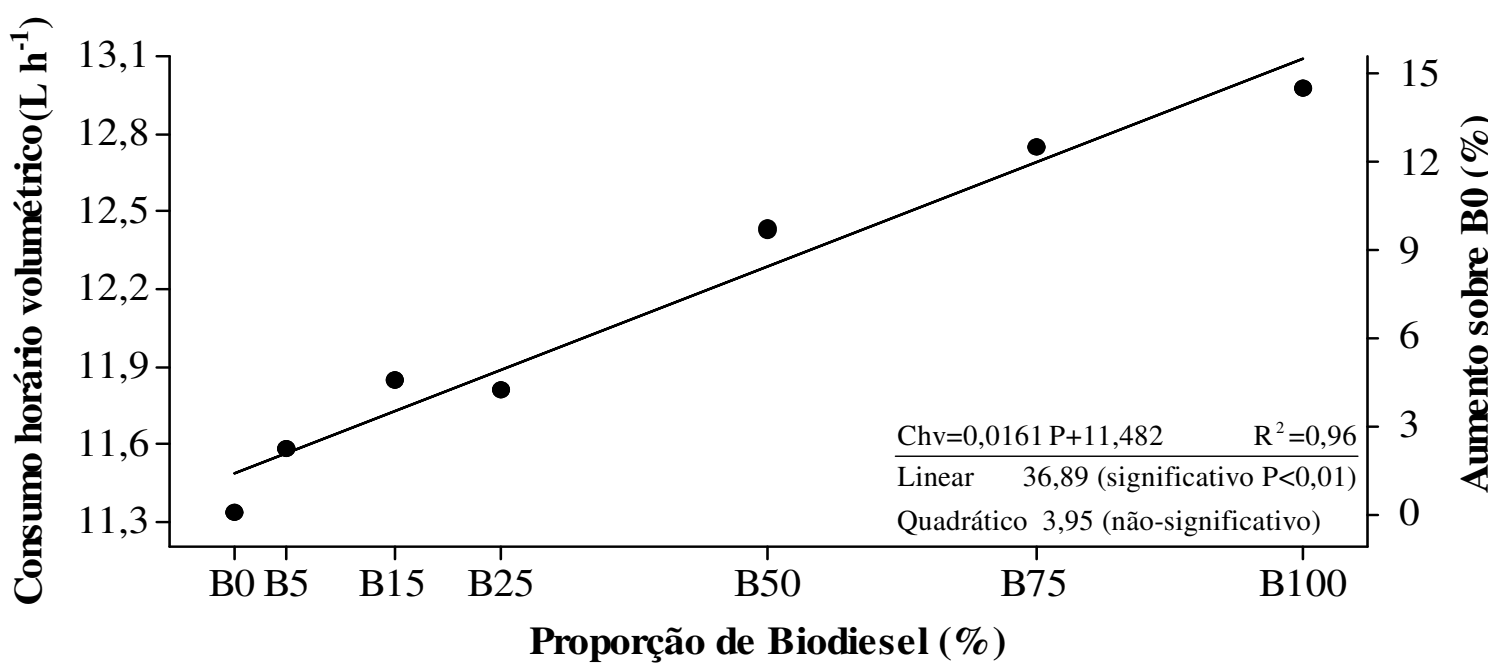

FIGURA 1. Consumo horário volumétrico em função da proporção de Biodiesel. Volumetric time consumption according to proportion of Biodiesel.

\section{Consumo horário ponderal}

Pela Tabela 1, nota-se que o diesel metropolitano apresentou menor consumo $(3,7 \%)$ em relação ao diesel interior, resultado esse em função da qualidade e da menor densidade desse combustível, uma vez que tais características influenciam no cálculo do consumo horário ponderal.

Para o consumo horário ponderal, ocorreu interação significativa entre os fatores proporção de Biodiesel e carga na barra, sendo o desdobramento apresentado na Tabela 2.

TABELA 2. Síntese do desdobramento da interação proporção de Biodiesel e carga na barra para consumo horário ponderal $\left(\mathrm{kg} \mathrm{h}^{-1}\right)$. Summary of the unfolding of the interaction of Biodiesel ratio and bar load for weight hourly consumption $\left(\mathrm{kg} \mathrm{h}^{-1}\right)$.

\begin{tabular}{cccrrrrc}
\hline Carga na & \multicolumn{7}{c}{ Proporção de Biodiesel } \\
\cline { 2 - 7 } Barra & B0 & B5 & \multicolumn{1}{c}{ B15 } & \multicolumn{1}{c}{ B25 } & \multicolumn{1}{c}{ B50 } & \multicolumn{1}{c}{ B75 } & \multicolumn{1}{c}{ B100 } \\
\hline $13 \mathrm{kN}$ & $8,00 \mathrm{Ba}$ & $8,21 \mathrm{Ba}$ & $8,45 \mathrm{Ba}$ & $8,49 \mathrm{Ba}$ & $9,42 \mathrm{Bb}$ & $9,72 \mathrm{Bbc}$ & $10,46 \mathrm{Bc}$ \\
$23 \mathrm{kN}$ & $10,42 \mathrm{Aa}$ & $10,81 \mathrm{Aab}$ & $11,31 \mathrm{Ab}$ & $11,53 \mathrm{Ab}$ & $12,43 \mathrm{Ac}$ & $13,53 \mathrm{Ad}$ & $14,20 \mathrm{Ad}$ \\
\hline
\end{tabular}

Médias seguidas de mesma letra maiúscula na coluna e minúscula na linha não diferem entre si, pelo teste de Tukey, a $5 \%$ de probabilidade.

Pela Tabela 2, analisando o consumo ponderal na linha (carga de $13 \mathrm{kN}$ ), verifica-se que até B25 não houve diferença; comparando B0 a B100, o consumo aumentou 30,7\% (Figura 2). Na carga de $23 \mathrm{kN}$, não houve diferença até B5; comparando B0 a B100, o consumo aumentou 36,6\%. 
Segundo CASTELLANELLI et al. (2008), LOPES (2006) e MURUGESAN et al. (2009), esse resultado é em função do menor poder calorífico e do aumento da densidade do Biodiesel em relação ao diesel. Analisando-se o consumo horário ponderal nas colunas (Tabela 2), verifica-se que a carga de $23 \mathrm{kN}$ apresentou maior consumo em relação à de $13 \mathrm{kN}$, em qualquer proporção de mistura, ressaltando-se que, em B0, o aumento foi 30,3\%, e em B100 foi 35,8\%, evidenciando-se o menor poder calorífico do Biodiesel.

Na Figura 2, apresenta-se a análise de regressão linear do consumo ponderal em função da proporção de mistura, e no eixo auxiliar, o aumento do consumo em percentagem com relação a B0. Devido à interação significativa entre os fatores proporção de Biodiesel e carga na barra, a regressão é apresentada para as duas cargas. Os resultados do presente trabalho condizem com os encontrados por CAMARA (2004), REIS et al. (2006) e GROTTA et al. (2008).



Proporcão de Biodiesel (\%)

FIGURA 2. Consumo horário ponderal em função da proporção de Biodiesel e carga na barra. Weight hourly consumption according to Biodiesel ratio and bar load.

\section{Consumo específico}

Pela Tabela 1, verifica-se que o trator consumiu 4,7\% menos combustível quando funcionou com diesel metropolitano; tal resultado deve-se à qualidade e à menor densidade do produto.

Observou-se, também, que, relacionando a proporção de Biodiesel, não houve diferença significativa até B25; todavia, comparando B0 a B100, o consumo aumentou 38,3\% (Figura 2). Esse aumento deve-se a dois fatores: a maior densidade e o menor poder calorífico do Biodiesel em relação ao diesel, de acordo com o apresentado por CASTELLANELLI et al. (2008), LOPES (2006) e MURUGESAN et al. (2009). Nos combustíveis utilizados, a densidade mínima encontrada foi $846,5 \mathrm{~g} \mathrm{~L}^{-1}$ para B0 (metropolitano), e a máxima $972,1 \mathrm{~g} \mathrm{~L}^{-1}$, para B100, proporcionando aumento de $125,6 \mathrm{~g} \mathrm{~L}^{-1}$ ou $14,8 \%$.

A carga de $23 \mathrm{kN}$ apresentou menor consumo em relação à de $13 \mathrm{kN}$, o inverso do que ocorreu para o consumo horário volumétrico e ponderal, reforçando a recomendação de utilizar a máxima capacidade de tração do trator. Na Figura 3, apresenta-se a análise de regressão linear do consumo volumétrico em função da proporção de mistura, e no eixo auxiliar, o aumento do consumo em percentagem com relação a B0. 


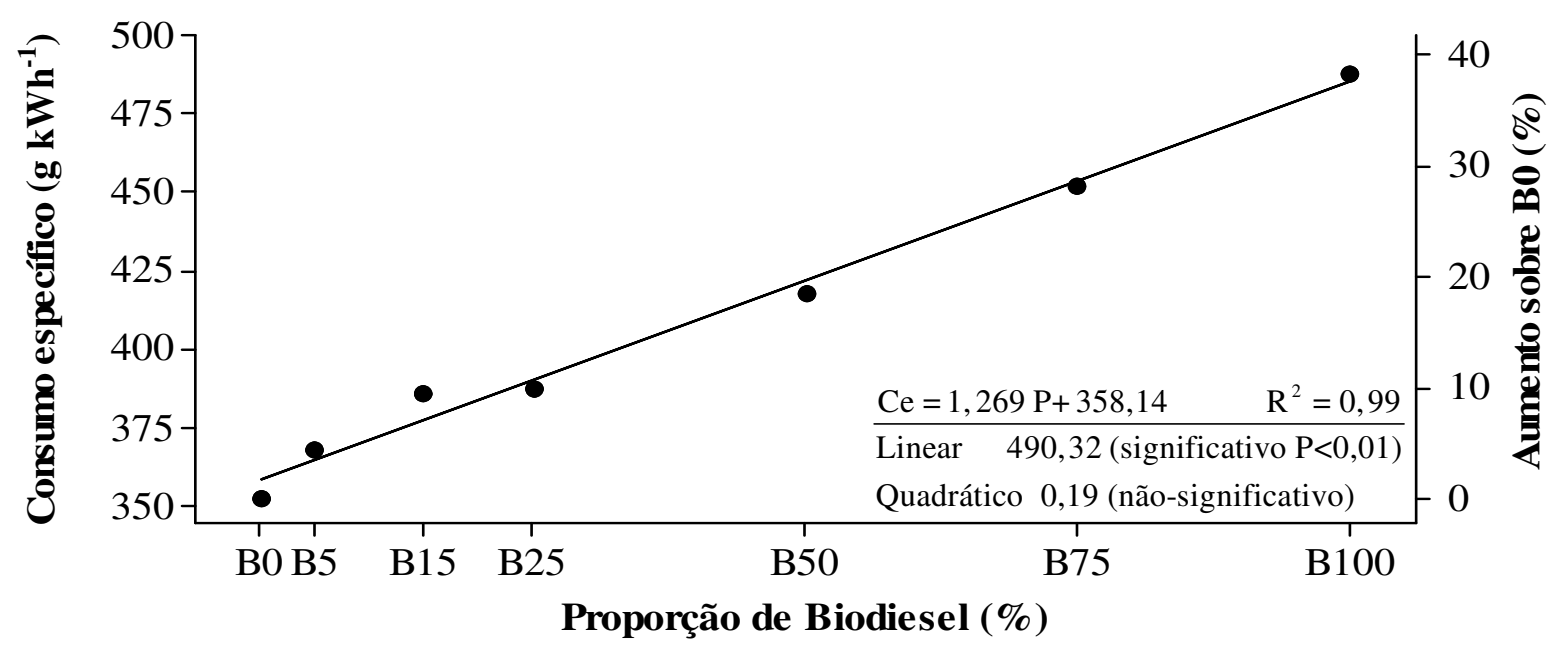

FIGURA 3. Consumo específico em função da proporção de Biodiesel. Specific consumption according to Biodiesel ratio.

Os resultados apresentados condizem com os encontrados por DORADO et al. (2003), LOPES (2006) e ANGELO (2006). O consumo específico é a forma mais utilizada para comparar tratamentos, pois leva em consideração a quantidade de combustível consumida, potência desenvolvida e densidade do produto.

\section{Opacidade}

Pela Tabela 1, observa-se que ocorreu interação significativa entre os fatores proporção de Biodiesel e diesel, sendo o desdobramento apresentando na Tabela 3.

TABELA 3. Síntese do desdobramento da interação diesel e proporção de Biodiesel para opacidade da fumaça $\left(\mathrm{m}^{-1}\right)$. Summary of the unfolding of the interaction of diesel and Biodiesel ratio for smoke opacity $\left(\mathrm{m}^{-1}\right)$.

\begin{tabular}{cccccccc}
\hline \multirow{2}{*}{ Diesel } & \multicolumn{7}{c}{ Proporção de Biodiesel } \\
\cline { 2 - 8 } & $\mathrm{B} 0$ & $\mathrm{~B} 5$ & $\mathrm{~B} 15$ & $\mathrm{~B} 25$ & $\mathrm{~B} 50$ & $\mathrm{~B} 75$ & $\mathrm{~B} 100$ \\
\hline Interior & $1,09 \mathrm{Aa}$ & $1,05 \mathrm{Aa}$ & $0,96 \mathrm{Abc}$ & $0,89 \mathrm{Acd}$ & $0,90 \mathrm{Acd}$ & $0,85 \mathrm{Ad}$ & $0,95 \mathrm{Abc}$ \\
Metropolitano & $0,94 \mathrm{Ba}$ & $0,93 \mathrm{Bab}$ & $0,92 \mathrm{Aab}$ & $0,86 \mathrm{Abc}$ & $0,88 \mathrm{Aabc}$ & $0,84 \mathrm{Ac}$ & $0,94 \mathrm{Aa}$ \\
\hline
\end{tabular}

Médias seguidas de mesma letra maiúscula na coluna e minúscula na linha não diferem entre si, pelo teste de Tukey, a $5 \%$ de probabilidade.

Pela Tabela 3, tanto na linha do diesel interior quanto no diesel metropolitano, verifica-se que a opacidade reduziu à medida que aumentou a quantidade de Biodiesel até a proporção de B75; comparando B0 a B100, a redução foi de 22,0 e 10,6\%, diesel interior e metropolitano, respectivamente. Porém, para os tipos de diesel a partir de B75, a opacidade voltou a aumentar, especificamente para o metropolitano, em que B100 foi semelhante a B0. Essa situação explica-se em função do alto índice de viscosidade do Biodiesel de mamona, como verificado por MAIA et al. (2006) e MURUGESAN et al. (2009), sendo tal característica a maior dificuldade para trabalhar com esse biocombustível.

$\mathrm{Na}$ Tabela 3, analisando as colunas, verifica-se que o diesel metropolitano apresentou melhor resultado em B0 e B5 (redução em relação ao diesel interior de 13,8 e 11,4\%, respectivamente). A partir de B15, o diesel interior iguala-se ao metropolitano, tornando-se notória a capacidade do Biodiesel em reduzir a opacidade. Os resultados encontrados concordam com os apresentados por MAZIERO et al. (2006) e MUÑOZ et al. (2004). O comportamento da opacidade em decorrência 
da proporção de Biodiesel é ilustrado na Figura 4, em que, no eixo auxiliar, é apresentada a redução da opacidade em percentagem com relação a B0.

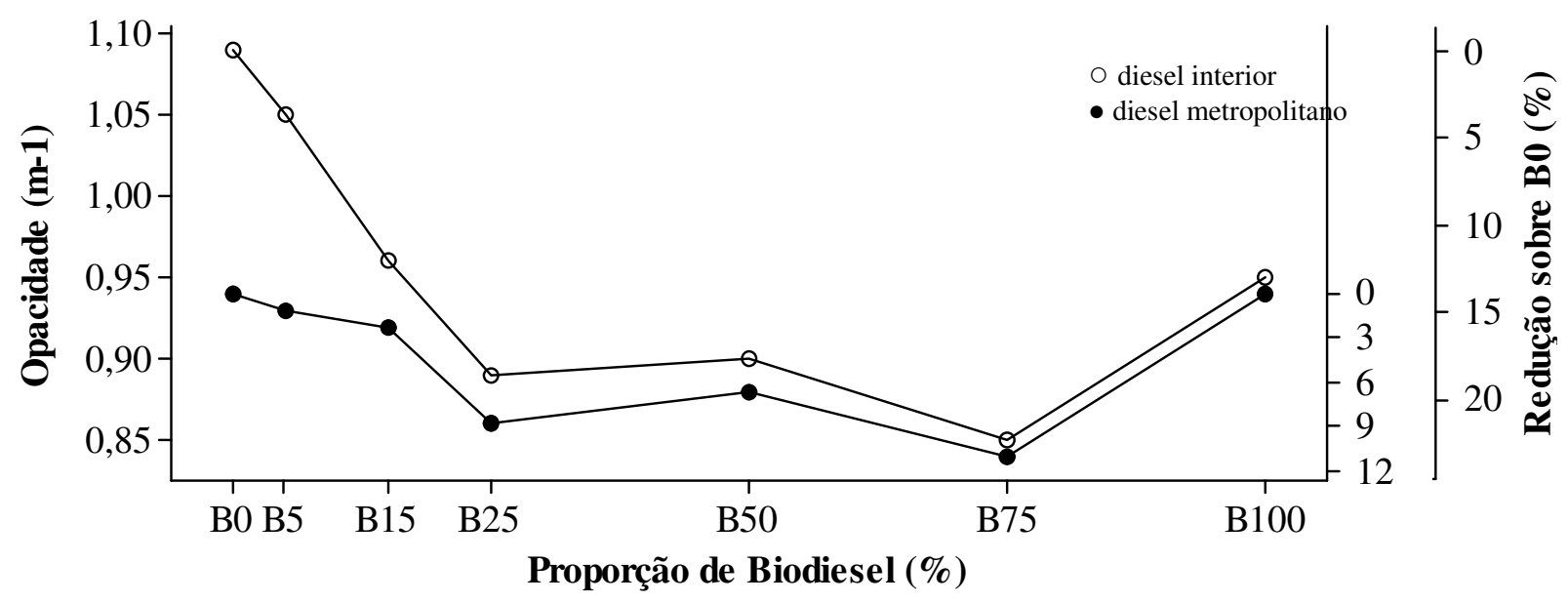

FIGURA 4. Opacidade da fumaça em função da proporção de Biodiesel e do tipo de diesel. Smoke opacity according to Biodiesel ratio and diesel type.

A redução da opacidade é representativa e favorável ao uso do Biodiesel, o que, em parte, é explicado pela ausência de enxofre no biocombustível. O enxofre compartilha o oxigênio disponível na fase tardia da combustão com o carbono resultante da queima parcial, em algumas condições de funcionamento do motor, aumentando a produção de material particulado (MAZIERO et al., 2006).

Pela diferença dos tipos de diesel explica-se a exigência de utilizar o metropolitano nos grandes centros urbanos, sendo essa ação ainda mais potencializada com a adição do Biodiesel, conforme disciplinada na Lei 11.097/2005.

\section{CONCLUSÕES}

O tipo de diesel influenciou no consumo de combustível e na opacidade da fumaça, com o metropolitano apresentando melhor resultado do que o de interior. À medida que se aumentou a proporção de Biodiesel (B0 até B100) nos dois tipos de diesel, o consumo também aumentou. A opacidade da fumaça reduziu com acréscimo de Biodiesel de mamona até B75 e cresceu em B100. Para a maior carga na barra de tração, ocorreu aumento no consumo horário volumétrico e ponderal, e o inverso, para o consumo específico.

\section{REFERÊNCIAS}

ANGELO, J. Análise da influência de diferentes misturas de biodiesel no desempenho e emissões de poluentes de um motor diesel agrícola. 2006. 159 f. Dissertação (Mestrado em Máquinas Agrícolas) - Escola Superior de Agricultura “Luiz de Queiroz", Universidade de São Paulo, Piracicaba, 2006.

BANZATTO, D.A.; KRONKA, S. N. Experimentação agrícola. Jaboticabal: FUNEP, 2006. 237 p.

BRASIL. Ministério de Minas e Energia. Agência Nacional de Petróleo. 2006a Anuário Estatístico Brasileiro do Petróleo, Gás Natural e Biocombustíveis 2006. Disponível em: <http://www.mme.gov.br>. Acesso em: 15 out. 2007.

BRASIL. Ministério de Minas e Energia. Agência Nacional de Petróleo. 2006b. Resolução ANP $N^{o}$ 15, de 17 de julho de 2006. Disponível em: <www.anp.gov.br/petro/legis>. Acesso em: 20 set. 2007. 
BRASIL. Ministério de Minas e Energia. 2007 Balanço Energético Nacional. Matriz Energética 1970 em diante. Disponível em: <http://www.mme.gov.br>. Acesso em: 15 out. 2007.

CAMARA, F.T. Desempenho de um trator agrícola com biodiesel etílico destilado. 2004. $54 \mathrm{f}$. Monografia (Trabalho de Graduação em Agronomia) - Faculdade de Ciências Agrárias e Veterinárias, Universidade Estadual Paulista, Jaboticabal, 2004.

CASTELLANELLI, M.; SOUZA, S.N.M.; SILVA, S.L.; KAILER, E.K. Desempenho de motor ciclo diesel em bancada dinamométrica utilizando misturas diesel/biodiesel. Engenharia Agrícola, Jaboticabal, v.28, n.1, p.145-153, 2008.

COSTA, F.C.; HOESCHL, H.C. Gestão do conhecimento na cadeia produtiva de Biodiesel. In: CONGRESSO DA REDE BRASILEIRA DE TECNOLOGIA DE BIODIESEL, 1., 2006, Brasília. Anais... Brasília: MCT/ABIPTI, 2006. p.30-34.

DORADO, M.P.; BALLESTEROS, E.; ARNAL, J.M.; GÓMEZ, J.; LOPEZ, F.J. Exhaust emissions from diesel engine fueled with transesterified waste olive oil. Spain Fuel, Valencia, v.82, n.1, p.1.311-1.315, 2003

EMBRAPA. EMPRESA BRASILEIRA DE PESQUISA AGROPECUÁRIA. Centro Nacional de Pesquisas de Solos. Sistema brasileiro de classificação de solos. Brasília, 1999. 412 p.

GROTTA, D.C.C.; LOPES, A.; FURLANI, C.E.A.; SILVA, R.P.; REIS, G.N.; CORTEZ, J.W. Biodiesel etílico de óleo residual de soja: desempenho de um trator agrícola na operação de gradagem. Acta Scientiaru Technology, Maringá, v.30, n.1, p.135-138, 2008.

LOPES, A. Biodiesel é fonte de energia renovável e barata para o País. Diário Oficial do Estado de São Paulo, São Paulo, 14 maio 2004. Seção 2, v.114, n.91, p.2.

LOPES, A. Biodiesel em trator agrícola: desempenho e opacidade. 2006. 158 f. Tese (LivreDocência em Agronomia) - Faculdade de Ciências Agrárias e Veterinárias de Jaboticabal,

Universidade Estadual Paulista, Jaboticabal, 2006.

MAIA, A.C.S.; TEIXEIRA, J.C.; LIMA, S.M.; FERREIRA, C.V.; STRAGEVITCH, L. Estudo do impacto da adição do biodiesel de mamona ao óleo diesel mineral sobre a propriedade viscosidade cinemática. In: CONGRESSO BRASILEIRO DE MAMONA, 2., 2006, Aracaju. Anais... 1 CDROM.

MANTOVANI, E.C.; LEPLATOIS, M.; INAMASSU, R.Y. Automação do processo de avaliação de desempenho de tratores e implementos em campo. Pesquisa Agropecuaria Brasileira, Brasília, v.34, n.7, p.1.241-1.246, 1999.

MAZIERO, J.V.G.; CORRÊA, I.M.; TRIELLI, M.A.; BERNARDI, J.A.; D’AGOSTINI, M.F. Avaliação de emissões poluentes de um motor diesel utilizando biodiesel de girassol como combustível. Engenharia na Agricultura, Viçosa - MG, v.14, n.4, 287-292, 2006.

MUÑOZ, M.; MORENO, F.; MOREA, J. Emissions of an automobile diesel engine fueled with sunflower methyl ester. Transactions of the ASAE, St. Joseph, v.47, n.1, p.5-11, 2004.

MURUGESAN, A.; UMARANI, C.; SUBRAMANIAN, R.; NEDUNCHEZHIAN, N. Bio-diesel as an alternative fuel for diesel engines. Renewable and Sustainable Energy Reviews, Uttaranchal, v.13, n.3 ,p.653-662, 2009.

PARENTE, E.J.S. Biodiesel: uma aventura tecnológica em um país engraçado. Fortaleza: Universidade Federal do Ceará, 2003. 65 p.

REIS, G.N.R.; LOPES, A.; FURLANI, C.E.A.; DABDOUB, M.J.; SILVA, R.P.; GROTTA, D.C.C.; CAMARA, F.T. Análise comparativa entre biodiesel filtrado e biodiesel destilado em trator agrícola. Revista de Ciências Agroveterinárias, Lages, v.5, n.2, p.149-155, 2006. 
SCHLOSSER, J.F.; CAMARGO, M.N.; MACHADO, P.R.M. Comportamento de um motor de ignição por compressão trabalhando com óleo diesel e gás natural. Engenharia Agrícola, Jaboticabal, v.24, n.3, 2004.

TECNOMOTOR. TECNOMOTOR ELETRÔNICA DO BRASIL. OPACER TM 133 - Opacímetro de amostragem: manual de operação. São Carlos, 2001. 26 p.

XIANOMING, L.; YUNSHAN, G.; SIJIN, W.; XIUKUN, H. An experimental investigation on combustion and emission characteristics of turbocharger engines fueled with blends of Biodiesel. In: SAE FUEL AND LUBRICANTS, 1., 2005, Rio de Janeiro. Anais... Rio de Janeiro: SAE, 2005. 1 CD-ROM. 